\title{
Perfil sociodemográfico e clínico de mulheres assistidas na consulta enfermagem ginecológica de uma localidade litorânea do Rio de Janeiro
}

\author{
Ricardo José Oliveira Mouta, M.Sc.*, Sandra Maria Oliveira Caixeiro-Brandão, M.Sc. ${ }^{* *}$, \\ Cristina Portela da Mota, M.Sc.***, Andréa Araújo Viana****
}

* Enfermeiro Obstétrico, Docente da Faculdade de Enfermagem da UERJ, Coordenação do Programa Saúde da Mulher do Município de Rio das Ostras/RJ, **Enfermeira de Saúde Pública, Docente da Universidade Iguaçu, ***Enfermeira obstétrica, Docente da Faculdade de EEAAC/UFF, ****Enfermeira Sanitarista e Obstétrica, Coordenação Programa Saúde da Mulher do Municipio de Rio das Ostras/RJ

\begin{abstract}
Resumo
Os objetivos do estudo foram descrever e discutir o perfil sociodemográfico e clínico das mulheres assistidas na consulta de enfermagem ginecológica em 2005 em uma unidade básica da baixada litorânea, do estado do Rio de Janeiro. Constituiu-se em estudo exploratório, descritivo e com abordagem quantitativa. A análise do perfil sociodemográfico permitiu concluir que a faixa etária de maior adesão ao programa é a de 21 até 30 anos, com percentual significativo de mulheres idosas; o principal estado civil é de mulheres casadas, emparelhando com o de mulheres sem cônjuge referido; as mulheres atendidas estão, em sua grande maioria, inseridas no mercado de trabalho, seguidas das profissionais do lar. Os principais resultados do perfil clínico através do Papanicolau apontaram para inflamação e metaplasia escamosa; NIC I e NIC II foram detectados um caso de cada. Foi detectado um percentual elevado de mulheres portadoras de agente infeccioso.
\end{abstract}

Palavras-chave: atenção primária à saúde, saúde da mulher, enfermagem.

\section{Abstract \\ Sociodemographic and clinical profile of women assisted in a gynecological nursing consultation of a city of Rio de Janeiro coastline}

The aim of this study was to describe and discuss the clinical and sociodemographic profile of women assisted at the gynecological consultation in 2005 of a health unit of the coast of Rio de Janeiro. It was an exploratory study with descriptive and quantitative approach. The analysis of the sociodemographic profile showed that the highest age group adherence to the program is from 21 to 30 years, with a significant percentage of older women; the main marital status is of married women, paired with women without husbands; most of the women who attended the program are inserted in the labor market, followed by housewives. The main results of the clinical profile through Papanicolau testing pointed out inflammation and 
squamous metaplasia; cervical intraepithelial neoplasia (CIN I and CIN II) were detected, one case each. A high rate of women with infectious agent was observed.

Key-words: primary health care, women health, nursing.

\section{Resumen}

\section{Perfil sociodemográfico y clínico de mujeres asistidas en la consulta de enfermería ginecológica en una ciudad costera de Río de Janeiro}

Los objetivos de este estudio fueron describir y analizar las características clínicas y el perfil sociodemográfico de mujeres que recibieron asistencia en la consulta de enfermería ginecológica en 2005 en una unidad básica de la ciudad costera del estado de Río de Janeiro. Se trata de un estudio exploratorio, descriptivo y cuantitativo. El análisis del perfil sociodemográfico indica que la edad de mayor adhesión al programa es de 21 a 30 años, con un porcentaje significativo de las mujeres de edad, el principal estado civil es la mujer casada, junto con el de las mujeres sin cónyuge; la mayoría de las mujeres atendidas están en el mercado de trabajo, seguidas por las que se ocupan de los trabajos en el hogar. Los principales resultados del perfil clínico a través del Papanicolau apuntaron para inflamación y metaplasia escamosa, NIC I y NIC II se detectaron un caso de cada. Se ha detectado un alto porcentaje de mujeres portadoras del agente infeccioso.

Palabras-clave: salud primaria, salud de la mujer, enfermería.

\section{Introdução}

A consulta de enfermagem foi legalmente estabelecida como atividade privativa do enfermeiro no ano de 1986, por meio da promulgaçáo da lei 7.498, que dispóe sobre o exercício profissional, sendo efetivamente regulamentada, em 1987, pelo Decreto 94.406. Caracteriza-se por contemplar o levantamento de problemas, prescrição e registro, através dos quais são identificados problemas de saúde-doença e implementadas açóes de enfermagem que possam contribuir para a promoção, prevençáo e recuperação da saúde da mulher [1].

Inicialmente foi implementada nos serviços de saúde, visando à promoção de saúde em gestantes de baixo risco e crianças reconhecidamente sadias, ou seja, do grupo materno-infantil, posteriormente foi sendo estendida a outros grupos sociais, tais como, adultos e idosos. Os grupos sociais que apresentam patologias previamente diagnosticadas, tais como, tuberculose, hanseníase, diabetes e hipertensão são inseridos na consulta de enfermagem através dos programas do Ministério da Saúde [1].

Nesse ínterim, ganha força a consulta ginecológica realizada pelo enfermeiro, que possui conhecimentos que valorizam as demandas culturais da mulher, a educação em saúde para o autocuidado e o perfil de multiplicador das ações em saúde [1].

A consulta de enfermagem ginecológica apresenta papel significativo na diminuição da morbimortalidade por câncer de colo de útero, haja vista a incidência de 19.260 mil (11\%) casos novos em
2006, perdendo apenas para o câncer de mama feminino. A mortalidade por câncer de colo de útero permaneceu estável no período [2].

Assim, ela vem se consolidando como modalidade assistencial nas unidades básicas de saúde e nos ambulatórios gerais e especializados, constituindo um avanço importante no combate e na prevenção das neoplasias de útero.

Nesse sentido, a consulta de enfermagem ginecológica [3], enquanto modalidade assistencial, foi implantada na unidade básica de saúde em um município da baixada litorânea, no ano de 2005, tendo por base açóes direcionadas aos aspectos preventivos relacionadas às neoplasias malignas, principalmente mama e útero. Sua atuação está intimamente ligada à educação em saúde das mulheres que buscam esse tipo de assistência.

Nessa unidade é realizado exame clínico ginecológico e coleta de material para exame citopatológico. São prestadas ainda orientaçóes sobre promoção da saúde, prevenção de doenças e diagnóstico precoce, trabalhando em conjunto com a equipe multiprofissional.

Com o início da realização da consulta de enfermagem em saúde da mulher pelo enfermeiro na unidade, tornou-se imprescindível conhecer o perfil sociodemográfico e os principais resultados do Papanicolau de uma amostra específica, para criar estratégias de qualificação do serviço, de acordo com as necessidades das mulheres.

As questóes que nortearam este estudo foram: Que mulher foi assistida pelo enfermeiro no ano 
de implantação da consulta de enfermagem ginecológica? Quais foram os principais resultados do Papanicolau encontrados no ano de 2005?

O detalhamento do perfil epidemiológico da mulher com vistas ao aprimoramento das açóes assistenciais e preventivas da doença e também da identificação das causas do câncer cérvico-uterino é fator determinante para iniciarmos uma queda sensível, porém importante nos índices de morbimortalidade por neoplasias de colo de útero [4].

A inserção do profissional enfermeiro na equipe multiprofissional da unidade, realizando a consulta de enfermagem ginecológica, trouxe um avanço para a categoria, pois a ocupação do espaço determina um crescimento do profissional.

A atuaçáo do enfermeiro nos programas de saúde, em especial no programa de saúde da mulher, é de suma importância, pois ele é um facilitador do acesso da cliente ao programa, bem como um articulador da equipe multiprofissional que detém em suas açóes a resolubilidade das necessidades de saúde da mulher em seu processo de trabalho.

Ante o exposto, os objetivos deste estudo são: descrever o perfil sociodemográfico e clínico das mulheres que foram assistidas na consulta de enfermagem ginecológica em uma unidade básica de saúde no ano de 2005 e discutir esse perfil utilizando-se das bases da epidemiologia descritiva.

\section{Material e método}

Trata-se de estudo observacional, descritivo, com abordagem quantitativa.

A coleta de dados foi realizada no período de março a abril de 2006, em uma unidade básica de saúde da baixada litorânea de um Município do Estado do Rio de Janeiro. O perfil das mulheres atendidas na unidade foi levantado pela técnica de análise documental.

Esse levantamento foi possível através da análise das informaçóes contidas no livro de registro de consulta de enfermagem ginecológica que é preenchido antes do início da consulta, do qual foram coletadas as variáveis: idade, estado civil e ocupação; e da análise do prontuário após a consulta, com os respectivos resultados do exame de Papanicolau.

A entrada no campo foi autorizada pelos responsáveis do Programa de Saúde da Mulher e o projeto foi aprovado pelo Comitê de Ética em Pesquisa, seguindo as recomendaçóes da Resolução 196/96, do Conselho Nacional de Saúde [5].
A população atendida nessa unidade de saúde é composta por mulheres que residem nas áreas rurais do município. A amostra foi composta por cem (100) mulheres cadastradas e assistidas no ano de 2005 pelo Programa de Saúde da Mulher e prevenção do câncer cervico-uterino, atendidas na consulta de enfermagem ginecológica. O prontuário das mulheres foi selecionado aleatoriamente, para evitar a interferência dos pesquisadores.

O perfil epidemiológico das mulheres foi construído a partir das variáveis: faixa etária, estado civil e família, ocupação e resultados dos exames de Papanicolau. A tabulação dos dados aconteceu com a utilização do programa Excel 2003. Para a apresentação dos dados, utilizou-se a análise estatística, com números absolutos dispostos em gráficos para a interpretação de cada variável levantada.

\section{Resultados e discussão}

\section{Perfil sociodemográfico das mulheres assistidas}

A variável idade está intimamente ligada à incidência de doenças e aos danos à saúde. A observação da distribuição etária da incidência de um agravo à saúde é muito valiosa para o levantamento de hipóteses sobre o aparecimento de doenças e nos fornece subsídios para orientar a elaboração de cuidados pertinentes ao agravo [6].

A faixa etária das mulheres estudadas variou de 11 a 90 anos, mostrando uma larga faixa de idade. A maior concentraçáo foi na faixa dos 21 a 30 anos (38\%), seguidos dos 31 a 40 e dos 41 a 50 anos (19\%), conforme observado na Figura 1.

Figura 1 - Distribuição das mulheres assistidas na consulta de enfermagem ginecológica em unidade básica de saúde no ano de 2005, segundo faixa etária.

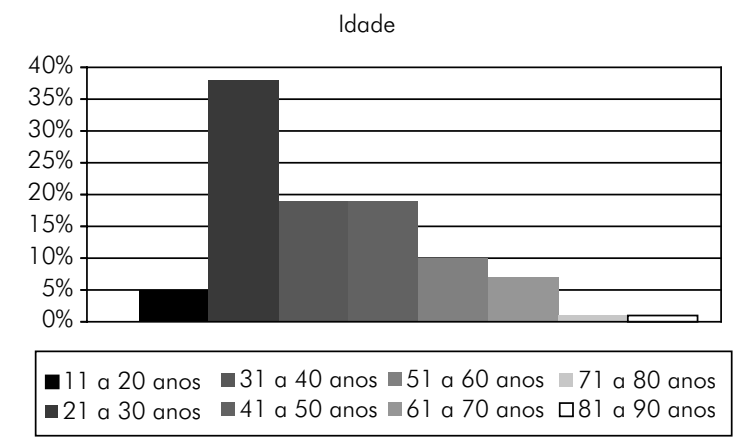

Fonte: Unidade básica de saúde baixada litorânea. 
O conjunto de mulheres que realizaram o exame Papanicolau quando estratificadas por faixa etária, apresentaram-se crescentes até 21-30 anos (38\%) e a partir daí com valores decrescentes até 81 anos ou mais.

O Ministério da Saúde, na década de 90, ampliou a faixa etária de mulheres com maior exposiçáo ao contágio, que antes era de 35 a 49 anos e passou para a faixa dos 25 a 59 anos, objetivando o planejamento de açóes para cobertura de mulheres dentro desta faixa etária [7].

Esses resultados evidenciam que a procura pelo exame de Papanicolau observada neste estudo confere com a tendência verificada na literatura. De acordo com um estudo realizado com 54 mulheres que buscaram o exame Papanicolau [8], a idade das mulheres variou de 17 a 36 anos; acima desta faixa etária, a procura pelo exame se reduziu à metade.

Observou-se, ainda, que a maior incidência das lesóes precursoras do câncer de colo de útero ocorreu dos 20 aos 39 anos, coincidindo com o período de vida fértil da mulher que, segundo o Ministério da Saúde [9], se inicia aos 10 anos e se estende até aproximadamente 49 anos. Notou-se que, acima da faixa etária reprodutiva, há menor procura das mulheres pelos serviços de saúde.

Observou-se também um quantitativo de 19 mulheres (19\%) fora da idade fértil que procuraram o serviço de enfermagem para a realizaçáo de preventivo ginecológico, um fato muito positivo, por tratar-se de uma regiáo rural e com maior número de idosas.

Sousa et al [10] destacam que a procura do serviço de saúde por idosas vem aumentando gradativamente, em virtude do envelhecimento da populaçáo brasileira, do aumento de idosos por unidades domiciliares e devido às necessidades específicas desse grupo social.

Esse dado anuncia um período de mudanças ou de transformação da população feminina idosa, que busca a promoçáo da saúde e a prevenção de doenças, e não somente o tratamento de patologias já instaladas.

$\mathrm{Na}$ consulta de enfermagem, o enfermeiro tem fundamental importância para as orientaçóes para o autocuidado da mulher em idade fértil, especialmente da mulher idosa. Com a demanda espontânea de mulheres pertencentes à terceira idade que procuram pela consulta de enfermagem ginecológica, alteram-se os sujeitos e, conseqüentemente, altera-se o cenário que necessita adequar- se para atender esse grupo, de acordo com suas características específicas.

Quanto ao estado civil, observou-se um percentual de $58 \%$ de mulheres casadas (58 mulheres), $36 \%$ eram solteiras (36 mulheres), $4 \%$ viúvas ( 4 mulheres) e $2 \%$ divorciadas ( 2 mulheres), conforme mostra a Figura 2. Numa análise geral, somandose os percentuais de mulheres solteiras, viúvas e divorciadas, nota-se um total de $42 \%$ de mulheres sem cônjuge.

Figura 2 - Distribuição das mulheres assistidas na consulta de enfermagem ginecológica em unidade básica de saúde no ano de 2005, segundo estado civil.

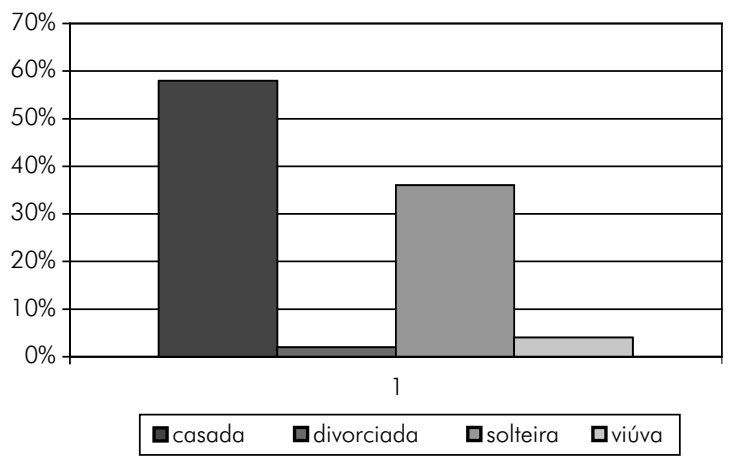

Fonte: Unidade básica de saúde baixada litorânea

Alguns autores [6,11-13] referem que o estado civil está associado à saúde das pessoas e das populaçóes. No entanto, no Brasil, este dado não foi confirmado, em virtude da escassez de pesquisas sobre a temática.

Segundo a literatura, a família confere certa proteção e segurança à mulher, favoráveis que refletem diretamente no nível de saúde da mesma. A vida a dois costuma trazer mudanças positivas no comportamento de saúde e sexual do casal. Em geral, alteram-se os riscos venéreos e a gravidez pode ser mais bem planejada; são conferidas estabilidade, qualidade de vida e proteçáo mútua, possibilitando ao casal lidar com os problemas da vida moderna, de maneira menos traumática [6].

Outra vertente ligada à uniáo estável e que pode trazer aspectos desfavoráveis à saúde da mulher é que, após o casamento, a mulher passa a ter vida sedentária, o que a predispóe a doenças crônicodegenerativas [6]; em geral, sentem-se menos vulneráveis às doenças sexualmente transmissíveis (DST's) e ao Vírus do Papiloma Humano (HPV), agente infeccioso mais importante no desenvolvimento de câncer do colo de útero, visto que o uso 
de preservativo (feminino ou masculino) entre os casais não é prática comum, por acreditarem que têm um parceiro fiel e confiável, o que nem sempre é fidedigno [14].

A recente preocupação das autoridades sanitárias com a feminização da Aids no Brasil, com as mulheres em idade fértil, donas de casa, mães de família e em união estável, vem reafirmar a vulnerabilidade dessas mulheres e a predisposição às DST's [15].

Em relação à AIDS, o quadro epidemiológico fica mais complexo a partir da década de 80 , quando ocorre a primeira notificaçáo da doença em uma mulher e, desde então, esse quadro vem aumentando de modo acelerado. A transmissáo heterossexual por contato sexual foi a mais provável, ocorrendo em mais de $80 \%$ dos casos. Parte expressiva das mulheres tem parceiros fixos e únicos, os quais teriam multiparceria ou seriam toxicômanos e/ou soropositivos [14].

A grande vulnerabilidade feminina ao HPV e às DST's ligada ao estado civil, à família e a conseqüente desvantagem na adoção de práticas preventivas, estaria baseada na assimetria de poder nas relaçóes de gênero, em especial no contato sexual [14].

Nesse sentido, durante a consulta ginecológica, a enfermeira deve traçar estratégias na orientação dessa mulher, desmistificando equívocos em relação ao estado conjugal, evitando a elevação das taxas de morbidade e mortalidade relacionadas ao estado civil.

Visto isso, destaca-se que, mesmo que $58 \%$ das mulheres deste estudo estejam em união consensual estável, elas têm procurado o serviço de saúde primário em busca de manter a saúde.

Em relação às mulheres solteiras ou sem cônjuge (42\%), observou-se que elas têm desenvolvido a capacidade de adaptação à condição de tornaremse independentes, assumindo responsabilidades de vida sem a figura do cônjuge, embora alguns autores afirmem que a presença do cônjuge pode ser muito valiosa para a segurança e estabilidade da mulher $[6,10]$.

As diferenças do perfil de morbidade e mortalidade relacionadas ao estado civil apresentam duas vertentes: uma conferida pela família, que representa um forte fator de proteção e propicia bem-estar, não só para a mulher, mas para todos os seus membros; outra conferida pela seleção para a união estável. Nesse último, as mulheres sadias tendem a manter uma união conjugal, enquanto as afetadas por patologias físicas ou mentais têm maior dificuldade em manter um companheiro e constituir família [6]. Há, portanto, um viés de seleção para a união estável, diretamente relacionada com a saúde da mulher.

A saúde da mulher também pode estar relacionada com o tipo de ocupaçáo que ela exerce, pois o ambiente e as condiçóes de trabalho podem deixá-la exposta a riscos durante boa parte de sua vida.

Entende-se por ocupação, o cargo, função, profissão ou ofício rotineiramente exercido [6].

Analisando a Figura 3, constatamos que 44\% das mulheres estudadas tinham ocupação exclusiva do lar (vida privada), 18\% eram empregadas domésticas, $13 \%$ estudantes, $6 \%$ agricultoras, $4 \%$ aposentadas e pensionistas, $3 \%$ professoras, vendedoras ou recepcionistas e $1 \%$ servidoras públicas, caseiras, garis, costureiras, cabeleireiras ou comerciante.

Em uma análise geral, observa-se que $52 \%$ das mulheres estáo no mercado de trabalho, desconsiderando os $4 \%$ de aposentadas e pensionistas que estão fora do mercado de trabalho.

Figura 3 - Distribuição das mulheres assistidas na consulta de enfermagem ginecológica em unidade básica de saúde no ano de 2005, segundo ocupação.

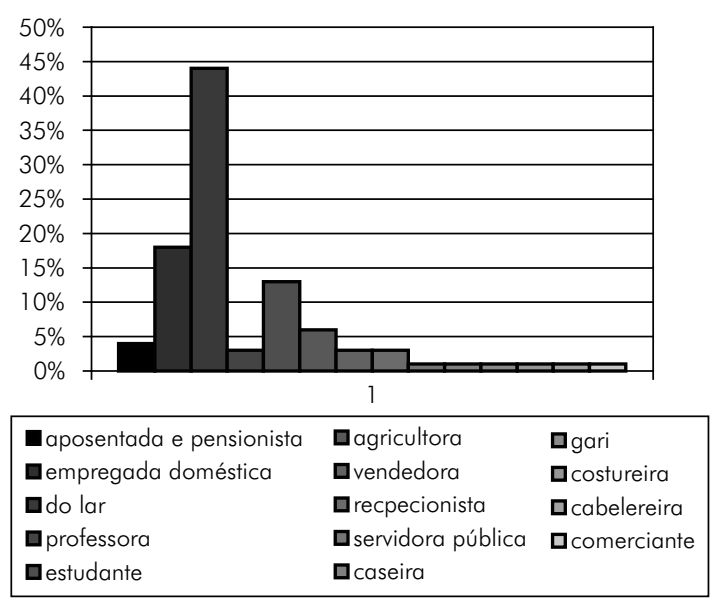

Fonte: Unidade básica de saúde baixada litorânea

O câncer relacionado ao trabalho é definido como aquele causado pela exposição durante o trabalho a agentes cancerígenos presentes no ambiente de trabalho. Os fatores de risco para esta patologia podem ser externos (ambientais) ou internos (hereditários), estando inter-relacionados e associados. Interagem de várias maneiras para dar início às alteraçóes celulares, sendo que, de $2 \%$ a $4 \%$ de todos os casos de câncer podem estar associados à vida pública da mulher [2]. 
Estudos demonstraram que na década de 70, menos de $20 \%$ das mulheres brasileiras trabalhavam fora de casa. Atualmente são $48 \%$ que, em geral são mais velhas, casadas e mães. $\mathrm{Na}$ década de 80 , apenas $20 \%$ eram esposas; atualmente são $50 \%[2]$.

A inserção da mulher no mercado de trabalho trouxe novos problemas para a classe. Elas mudaram o estilo de vida, ficando expostas a fatores de risco extrínsecos, tais como, exposição ocupacional, poluição ambiental, uso do tabaco, álcool, hábitos alimentares inadequados, inatividade física, entre outros [2].

A atividade física regular, por exemplo, tem papel protetor em relação ao câncer de mama e endométrio. Ela reduz o risco da mulher desenvolver câncer em $20 \%$ a $40 \%$, devido à diminuição dos níveis dos hormônios femininos. Além disso, influencia diretamente no peso corporal da mulher. O excesso de peso (sobrepeso e/ou obesidade) está associado ao risco aumentado de desenvolver câncer de mama e endométrio na pós-menopausa [2].

A entrada das mulheres no mercado de trabalho não as desobrigou dos cuidados com a casa, o cônjuge e os filhos, o que acaba resultando em sobrecarga de tarefas e, conseqüentemente, efeitos negativos sobre sua saúde [14].

A maioria dos companheiros não auxilia a mulher nas tarefas do lar que exigem, em média, 30 horas semanais. Quando essas mulheres trabalham fora de casa, soma-se essa carga horária à jornada de 44 horas de trabalho, perfazendo um total de quase 75 horas semanais. Esta situação culmina em conflitos morais, transtornos na vida sexual do casal, desentendimentos com familiares e sentimentos de culpa, afetando diretamente a saúde física e mental da mulher [16].

Os critérios de avaliação dos agravos da dupla jornada de trabalho à saúde da mulher, ainda não contemplam as especificidades do trabalho feminino, ou seja, os instrumentos teóricos e metodológicos utilizados para este fim ainda são os mesmos utilizados para a população masculina que apresenta características de trabalho diferenciadas ao das mulheres. As informaçóes específicas voltadas para avaliar o conjunto de trabalhadoras ainda sáo insuficientes. Ainda assim, sem um refinamento adequado, estes estudos epidemiológicos apontam para estresse, problemas mentais, osteomusculares e fadiga [14].
Outro fator de risco muito importante e pouco divulgado para o carcinoma de colo de útero é a relação deste com a ocupação do companheiro. Segundo a classificação social inglesa, que distribui as ocupaçóes em cinco níveis - profissionais liberais (classe I), de nível médio (classe II), trabalhador qualificado (classe III), semiqualificado (classe IV) e náo-qualificado (classe V)-, a mortalidade para esse tipo de câncer, por exemplo, é de $25 \%$ para companheiros que tenham por ocupação ser motorista trabalhando no meio rural (trabalhador semiqualificado) e de $263 \%$ para companheiros que são marinheiros, também trabalhador semiqualificado [6]. Esta relação é inversamente proporcional, ou seja, quanto maior o nível social do trabalhador (nível cinco), menor é a exposição da mulher ao carcinoma de colo uterino.

Com a sobrecarga de trabalho, a mulher tem pouco tempo para cuidar da sua saúde individual, procurando o serviço de saúde somente após o aparecimento de agravos já instalados. Nessas condiçóes, o trabalho apresenta-se como fator contributivo, provocador ou agravador de patologias preexistentes, principalmente se a mulher tem forte tendência hereditária para o câncer de útero, se está exposta a outros fatores de risco e se náo procura rotineiramente o serviço básico de saúde para a realização do preventivo ginecológico.

\section{Perfil clínico de acordo com o resultado do Exame Papanicolau}

Nessa categoria, analisa-se os principais resultados do exame de Papanicolau (Exame Preventivo de Câncer do Colo Uterino) e como estes podem influenciar a saúde ginecológica da mulher.

A Figura 4 apresenta a distribuição dos resultados dos exames de Papanicolau das mulheres deste estudo. Observa-se que 29\% (29) das mulheres são portadoras de inflamaçáo uterina, 19\% (19) têm metaplasia escamosa, 12\% (12) algum tipo de bacilo, 11\% (11) lactobacilos, 7\% (07) cocos, 6\% (06) gardenerela, além de outros agentes como cândida sp., flora mista e atrofia, células anormais, trichomonas, neoplasia intra-epitelial cervical grau I (NIC I) e grau II (NIC II), Herpes vírus e flora inaparente.

Somente 03 (3\%) mulheres não apresentaram resultado positivo para qualquer tipo de microorganismo. 
Figura 4 - Distribuição dos resultados dos Exames Papanicolau das mulheres assistidas na consulta de enfermagem ginecológica em unidade básica de saúde no ano de 2005.

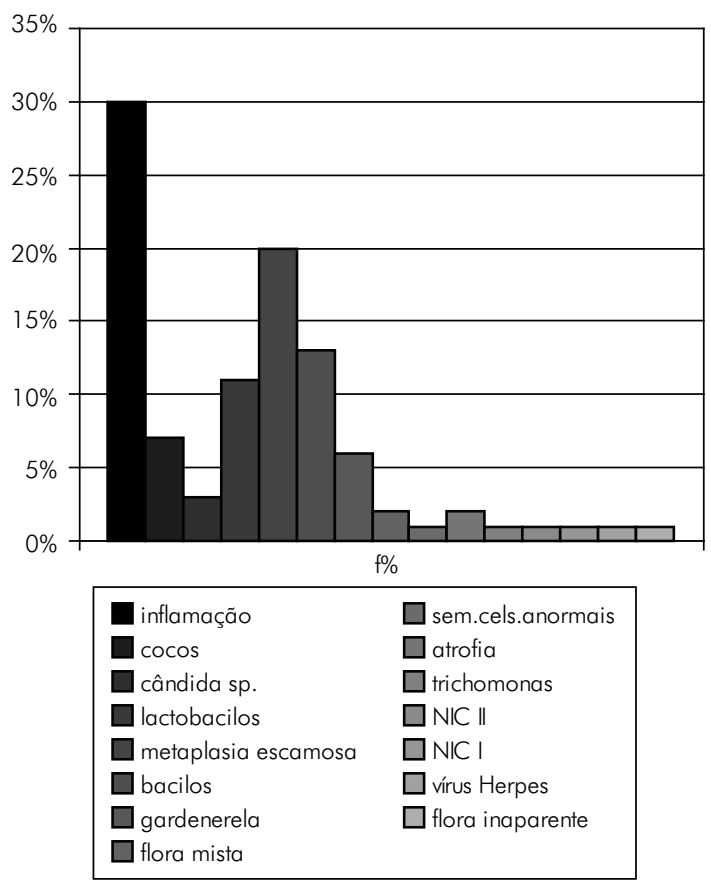

Fonte: Unidade básica de saúde baixada litorânea

A inflamação foi elemento de maior incidência (29\%) entre as mulheres deste estudo. Este percentual é preocupante, visto que a inflamação do colo uterino está entre as principais causas de câncer uterino.

Alguns vírus, bactérias e parasitas associados a infecções crônicas estão intimamente ligados no processo de desenvolvimento do câncer. Uma inflamaçáo crônica causada por agente infeccioso pode alterar o comportamento das células infectadas, comprometendo o sistema imune e aumentando o risco de câncer [2].

Segundo a International Agency for Research on Cancer (IARC), o principal agente envolvido no carcinoma cervical é o Papilomavírus Humano (HPV). É um vírus sexualmente transmissível, associado a diversos tipos de câncer. Os tipos mais freqüentes são o HPV 16 e 18, relacionados com $70 \%$ dos casos de cânceres. O HPV é considerado o agente infeccioso mais importante no desenvolvimento do câncer, sendo atribuído a ele $100 \%$ dos casos de câncer do colo do útero e 5,2\% dos casos de câncer no mundo, para ambos os sexos, dentre os quais se destacam os cânceres anogenitais, vaginais, vulvares, penianos, anais, de boca e orofaringe [2].
Outro dado relevante foi o aparecimento de atipias celulares escamosas (metaplasia escamosa), em $19 \%$ das mulheres estudadas e da fase pré-invasora, NIC I e NIC II, cada um em 1\% das mulheres.

As atipias celulares são células atípicas, de significado indeterminado, podendo ser escamosas ou glandulares. As escamosas, possivelmente não são neoplásicas. Entretanto, quando encontradas, não se descarta a lesão intra-epitelial de alto grau (NIC III) [2].

Já o NIC I é uma anormalidade do epitélio escamoso cervical em terço proximal da membrana e o NIC II compromete até dois terços da espessura do epitélio. Embora consideradas neoplásicas, NIC I e NIC II são lesôes reversíveis se identificadas e tratadas precocemente [2].

Considerando o total de casos de inflamaçáo, de atipias celulares escamosas, NIC I e NIC II, teremos um total de $50 \%$ de mulheres que apresentaram elementos significativos e merecedores de atenção por parte das autoridades e dos profissionais de saúde da unidade estudada. Mesmo não tendo se considerado os demais resultados (47\%) não menos importantes, é evidente a necessidade de se implementar medidas sanitárias de prevenção para infecções uterinas.

Nesse aspecto, o enfermeiro contribui promovendo o controle dos fatores de risco do câncer do colo do útero e das doenças sexualmente transmissíveis, organizando açôes para aumentar o número de mulheres que se submetem ao Papanicolau, rastreando mulheres com Papanicolau alterado e orientando para o autocuidado eficaz para a prevenção do câncer.

Os resultados deste estudo mostram o quão importante é a educação em saúde praticada na consulta de enfermagem ginecológica, bem como a participação do enfermeiro como membro da equipe multidisciplinar para discutir as medidas de promoção da saúde e de prevenção do carcinoma uterino, tanto no nível individual quanto no coletivo.

Considerando que a prevenção é a antecipaçáo ao acontecimento, impedindo que um fato ocorra ou mesmo que tenha continuidade, as atitudes e açóes preventivas ao câncer cérvico-uterino devem ser abrangentes, a fim de evitar o início do processo de cancerização ou mesmo a interrupção da evolução de uma lesão pré-maligna, para maligna. Para tanto, durante a consulta de enfermagem ginecológica, o enfermeiro utilizará recursos diagnósticos disponíveis para prevenir os fatores de risco para o câncer de colo de útero. 


\section{Conclusão}

A análise do perfil sociodemográfico das mulheres assistidas por enfermeiros na consulta de ginecologia permitiu concluir que a faixa etária de maior adesão ao programa é a de 21 até 30 anos, com percentual significativo de mulheres idosas; houve predomínio de mulheres casadas, emparelhando com o número de mulheres sem cônjuge referido; as mulheres atendidas estáo no mercado de trabalho, seguidas das profissionais do lar.

Os principais resultados do perfil clínico apontam para inflamação e metaplasia escamosa. Foi detectado um percentual elevado de mulheres portadoras de algum tipo de agente infeccioso.

Durante a consulta de enfermagem essas mulheres recebem orientaçóes diversas, principalmente quanto às questóes relativas à sexualidade feminina e masculina.

As ações são direcionadas para as mulheres com doenças sexualmente transmissíveis, no climatério e menopausa, portadoras de agravos ginecológicos com fatores de risco para o câncer de mama e de útero, detecção de vaginoses, com início do tratamento ou encaminhamento a serviços especializados.

O planejamento e a implementação da consulta de enfermagem ginecológica na unidade básica de saúde apontam a prevenção e o diagnóstico precoce como benefício social e econômico para o município.

Como proposta, destaca-se a realização de outros levantamentos nos anos subseqüentes à implantaçáo da consulta de enfermagem ginecológica, comparando os dados anteriores aos atuais, com o objetivo de direcionar a assistência prestada às reais demandas das mulheres da baixada litorânea.

\section{Referências}

1. Caixeiro SMO. O valor da consulta de enfermagem, como etapa do processo de assistência, à saúde da comunidade para o enfermeiro. Enfermagem Brasil 2006;5(4): 200-6.
2. Instituto Nacional de Câncer. Açóes de enfermagem para o controle do câncer: uma proposta de integraçáo ensino-aprendizagem. Instituto Nacional de Câncer. $3^{\mathrm{a}}$ ed. Rio de Janeiro: INCA; 2008. 628p.

3. Freitas SLF, Arantes SL, Barros SMO. Atuação da enfermeira obstetra na comunidade Anhanguera, Campo Grande (MS), na prevenção do câncer cérvico-uterino. Rev Latinoam Enfermagem 1998;6(2):57-64.

4. Rouquayrol MZ, Almeida Filho N. Epidemiologia e saúde. 6a ed. Rio de Janeiro: Medsi; 2006.

5. Ministério da Saúde. Conselho Nacional de Saúde. Diretrizes e normas regulamentares de pesquisa envolvendo seres humanos. Resolução no 196/96 Brasília: Ministério da Saúde; 2000.

6. Pereira MG. Epidemiologia. Teoria e prática. Rio de Janeiro: Guanabara Koogan; 2001.

7. Ministério da Saúde. Controle do câncer do colo uterino: Programa Nacional de Controle do Câncer do Colo Uterino. Secretaria Executiva. Brasília: Ministério da Saúde; 2001.

8. Pelloso SM, Carvalho MDB, Higarashi IH. Conhecimento das mulheres sobre o câncer cérvico-uterino. Acta Sci, Health Sci 2004;26(2):319-24.

9. Ministério da Saúde. Idade de vida fértil. [citado 2009 Jul 10]. Disponível em URL: http://www.portal.saude. gov.br/.

10. Sousa AI, Silver LD. Perfil sociodemográfico e estado de saúde auto-referido entre idosas de uma localidade de baixa renda. Esc Anna Nery Rev Enferm 2008;12(4): 706-16.

11. Berkson J. Mortality and marital status: reflections on the derivation of etiology from statistics. Am J Public Health Nations Health 1962;52:1318-29.

12. Bowling A. Mortality and bereavement: a review of the literature on survival periods and factors affecting survival. Soc Sci Med 1987;24(2):117-24.

13. Goodwin JS, Hant WC, Key CR, Samet JM. The effect of marital status on stage treatment and survival of cancer patients. JAMA 1987;258:3125-30.

14. Leão EM, Marinho LFB. Saúde das mulheres no Brasil: subsídios para as políticas públicas de saúde. Revista Promoção da saúde: saúde da mulher brasileira [online]. [citado 2009 Jul 10]. Disponível em URL: http://bvsms.saude.gov.br/bvs/is_digital/is_0303/pdfs/ IS23(3)079.pdf

15. Vieira BDG, Mota CP, Caixeiro SMO, Alves VH. Refletindo sob à luz da enfermagem: a feminização da Aids no campo de trabalho. Anais do $58^{\circ}$ Congresso Brasileiro de Enfermagem. Salvador; 2006.

16. Instituto Nacional de Câncer. Estimativa 2005: incidência de câncer no Brasil. Secretaria de Atençáo à Saúde. Instituto Nacional de Câncer. Rio de Janeiro: INCA; 2004. 